\title{
Metronidazole therapy for periodontitis
}

W. J. Loesche, E. Schmidt, B. A. Smith, R. Caffessee and J. Stoll

University of Michigan, School of Dentistry, Ann Arbor, Michigan, U.S.A.
Loesche WJ, Schmidt E, Smith BA, Caffessee R and Stoll J: Metronidazole therapy

for periodontitis. Journal of Periodontal Research 1987: 22: 224-226.
Accepted for publication September 26, 1986
Considerable data have accumulated which suggest that some or most forms of periodontal disease are specific, albeit chronic, bacteriological infections $(1,2)$. In most instances the plaque flora removed from inflamed sites are dominated by anaerobic organisms such as spirochetes, bacteroides and eubacterium species (1-4). In some instances, the microaerophilic organism Actinobacillus actinomycetemcomitans (Aa) has been associated with periodontal pathology $(5,6)$. These findings indicate that treatments aimed at controlling periodontal disease could contain an antimicrobial component directed to the reduction, if not the elimination of the above periodontopathic organisms in or from the plaque.

The choice of which antimicrobial agent to use would depend on the bacterial nature of the plaque infection and as to whether the agent is to be delivered systemically and/or locally (1). Agents such as metronidazole and clindamycin would seem to be indicated when an anaerobic infection has been documented, whereas tetracyclines would be indicated where an Aa infection has been established $(6,7)$. Alternatively, broad spectrum antiseptics such as chlorhexidine can be delivered locally to the pocket either via irrigation (8) or slow release delivery systems (9).

The efficacy of antimicrobial agents can best be evaluated by the inclusion of placebo groups and the use of a double blind design. In a double blind (DB) design all patients could receive the required mechanical debridement, so as to fulfill the ethical considerations for treatment, and then randomly receive either placebo (positive control) or antimicrobial medications (experimental). The antimicrobial treatments need only be of short duration, say 1 or $2 \mathrm{wk}$, because this length of treatment, provided an appropriate dose is prescribed and taken by the patient, is sufficient to change the composition of the plaque flora in most instances $(1,10)$.

Tetracyclines and metronidazole (Met) have been the agents most widely studied in the treatment of periodontitis, but only metronidazole has been evaluated using a DB design $(8,10)$. In these studies, Met significantly increased apparent attachment gain and reduced probing depths in the deepest probing sites relative to mechanical debridement (10), or irrigation with chlorhexidine (8). This suggested that the systemically delivered Met was able to reduce or eliminate the anaerobic infection at the base of the pocket in ways that were not routinely achievable with mechanical debridement or chlorhexidine irrigation.

The success of Met in both reducing probing depth and in increasing apparent attachment raised the possibility that Met therapy could reduce the surgical needs of the patient with advanced periodontitis. This was assessed using a DB design (10) and measuring the changes in those clinical parameters, i.e. appearance of tissue, x-ray changes, probing depths, apparent attachment gain, that resulted in the clinical decision to perform surgery. In this regard each tooth that required some form of periodontal surgery on one or more of its aspects was considered a surgical unit. The number of teeth requiring surgery at the onset of the study and again at the conclusion of the root surface debridement was determined for each patient by the same periodontist. This individual was not aware of the treatment groups that the patients were in, was not involved in the treatment procedures, and on the second examination was not provided access to the results of his first examination. Thus, while these surgical decisions had a large subjective component and maybe were unique to this examiner, they were obtained under strictly blinded conditions and reflected the reality of the patients' subsequent treatment, i.e. they did or did not receive surgery based upon this individual's decision. It was assumed that any changes in surgical needs between the first and second examinations that resulted from examiner error would be equal in both groups and would not mask a treatment effect if one existed.

Patients were selected for the study on the basis of having at least 5 surgical units. The patients randomly assigned to the Met group initially required 19.2 units of surgery and those assigned to the placebo group required 16.6 units of surgery. When reexamined after the completion of scaling and root planing, the Met patients required 10.7 units and the placebo patients required 13.6 units. The reduction of 8.5 units in the Met group was significant (paired $t$ test, $\mathrm{p}=$ $0.05)$, whereas the reduction in the placebo group was not. Covariate analysis showed that the difference of 5.5 units between the two groups was significant $(p=0.05)$.

These data indicate that $1 \mathrm{wk}$ of Met therapy, when superimposed upon rigorous mechanical debridement can significantly reduce the surgical needs of the patient when compared to placebo therapy plus rigorous mechanical debridement. This reduction had immediate cost and comfort benefits for the patients and would seem to be an important outcome of Met therapy. However, it can be argued that this surgical reduction was only surgery delayed and that these sites would eventually need surgery. This issue was addressed by maintaining the DB nature of the study and having the same clinician reexamine the patients during the recall visits.

The first such annual reexamination, which occurred about 27 months after the taking of the medication, about 20 months after the second treatment planing and 12 months after completion of the required surgery, showed that the 
placebo patients required on average 0.3 surgical units and 5.8 teeth that needed to be reinstrumented, whereas the Met patients required an average of 0.2 surgical units and 3.6 teeth that needed to be reinstrumented. Apparently, the beneficial effects of the Met were sustained as there was no increase in treatment needs in the Met group relative to the placebo group.

These clinical findings were also evaluated by the compliance pattern that had been observed some 27 months earlier by the microscopic monitoring of the spirochetal levels and proportions in the plaque. The study had been designed so that debridement was performed on only one quadrant during the period of drug administration, so that it was possible to assess the immediate effects of debridement, Met plus debridement, Met and no treatment upon the plaque levels and proportions of spirochetes, i.e. organisms uniquely sensitive to metronidazole (11). Thus patients who received Met and in whom the plaque levels and/or proportions of spirochetes did not ap-

Table 1. Compliance monitoring of systemic metronidazole as judged by change in proportions of plaque spirochetes - half mouth design

Metronidazole Placebo

No Debridement Debridement No Debridement Debridement

Frequency Distribution of \% Change of Spirochetes within Individual Sites

\begin{tabular}{lrrrr}
\hline Spirochetes not detected & 4 sites & 7 sites & 1 sites & 7 sites \\
$\downarrow>20 \%$ & 5 sites & 7 sites & 2 sites & 4 sites \\
$\downarrow 6-19 \%$ & 4 sites & 7 sites & 6 sites & 4 sites \\
$\pm 5 \%$ & 2 sites & & 5 sites & 2 sites \\
$\uparrow 6-19$ & 2 sites & & 4 sites & 5 sites \\
$\uparrow \quad 1$ sites & & 1 sites & \\
\hline Compliance $^{a}$ & 13 sites & 16 sites & 9 sites & 15 sites \\
Noncompliance $^{a}$ & 5 sites & 0 sites & 10 sites & 7 sites \\
\hline
\end{tabular}

a) compliance assigned when $\%$ spirochetes decreased $6 \%$ or more; noncompliance assigned when $\%$ spirochetes exhibited no change or increased.

$\downarrow$ decreased by

$\uparrow$ increased by.

Table 2. Change in spirochetal levels in plaque as a result of local metronidazole treatment

\begin{tabular}{|c|c|c|}
\hline \multirow[b]{2}{*}{ Spirochetes/hpf } & \multicolumn{2}{|c|}{ Frequency Distribution $(\mathrm{n}=25)$} \\
\hline & Before Treatment & After Treatment \\
\hline$>10$ & 10 sites & 0 sites \\
\hline $1-10$ & 14 sites & 3 sites \\
\hline$<1$ & 1 sites & 22 sites \\
\hline \multicolumn{3}{|l|}{ Chi-square $\mathrm{p}<0.01$} \\
\hline $\begin{array}{l}\text { ave spirochetes/hpf } \\
\text { ave } \% \text { spirochetes }\end{array}$ & $\begin{array}{c}12.6 \pm 2.7 \\
54.2 \pm 4.2 \leftarrow\end{array}$ & $\begin{array}{l}\rightarrow 0.5 \pm 0.2 \\
\rightarrow 13.6 \pm 3.7\end{array}$ \\
\hline
\end{tabular}

a) an ethylcellulose film (N7F) containing 20\% metronidazole was placed into each of 25 pockets for periods of 2 to $7 \mathrm{~d}$.

b) paired $t$ test. directly into the periodontal pocket $(9$, $12,13)$. We have evaluated an ethylcellulose film containing 20\% Met for its ability to reduce the levels and proportions of spirochetes. These films were formulated with the N7F formulation of ethylcellulose because this polymer is FDA-approved as a food additive, and would not be perceived as causing any untoward effects if swallowed. These brittle films were not well retained within the pocket, but even so they were effective in reducing plaque levels and proportions of spirochetes (Table 2). Films made with the N100 ethylcellulose have been retained for longer periods. Thus the problem of noncompliance would seen to be solved by the placement of films into the pocket which release Met in a slow but continuous fashion.

These findings from DB studies indicate that the use of Met in conjunction with scaling and root planing provides a significantly better clinical result, as judged by reduced need for surgery, than the use of scaling and root planing alone. Patient noncompliance with systemic medication can confound the results, but this problem may be resolved by the placement of slow release films containing Met directly into the pocket.

\section{Acknowledgments}

This study was supported by grant DE06030 from the National Institute of Dental Research.

\section{References}

1. Loesche, W. J. 1976. Chemotherapy of dental plaque infections. Oral Sci Rev 9: 63-107.

2. Savitt, E. D. \& Socransky, S. S. 1984 Distribution of certain subgingival microbial species in selected periodontal conditions. J Periodont Res 19: 111-123.

3. Loesche, W. J., Syed, S. A., Schmidt, E. \& Morrison, E. C., 1985. Bacterial profiles of subgingival plaques in periodontitis. J Periodontol 56: 447-456.

4. Moore, W. E. C., Holdeman, L. V., Cato, E. P., Smibert, R. M., Burmeister, J. A. \& Ranney, R. R. 1983. Bacteriology of moderate (chronic) periodontitis in mature adult humans. Infect Immun 42: 510-515.

5. Tanner, A. C., Socransky, S. S. \& Goodson, J. M. 1984. Microbiota of periodontal pockets losing crestal alveolar bone. J Periodont Res 19: 279-291.

6. Zambon, J. J. 1985. Actinobacillus actinomycetemcomitans in human periodontal disease. $J$ Clin Periodontol 12 $1-20$. 
7. Lindhe, J. 1982. Treatment of localized juvenile periodontitis. Chapter in HostParasite Interaction in Periodontal Disease. Genco, R. J. and S. E. Mergenhagen, eds., Amer. Soc. for Microbiol., Washington, D.C., pp. 382-394.

8. Joyston-Bechal, S., Smales, F. C. \& Duckworth, R. 1984. Effect of metronidazole on chronic periodontal disease in subjects using a topically applied chlorhexidine gel. $J$ Clin Periodontol 11: 53-62.

9. Soskolne, A., Golomb, G., Friedman, M. \& Sela, M. 1983. New sustained-release dosage form of chlorhexidine for dental use. J Periodont Res 18: 330-336.

10. Loesche, W. J., Syed, S. A., Morrison, E. C., Kerry, G. A., Higgins, T. \& Stoll, J. 1984. Metronidazole in periodontitis. I. Clinical and bacteriological results after 15 to 30 weeks. $J$ Periodontol 55 325-335.

11. Davies, R. M. \& Stirland, R. M. 1970. The in vitro sensitivity of oral spirochetes to metronidazole. J Periodont Res 5: 183-186.

12. Addy, M. \& Langeroudi, M. 1984. Comparison of the immediate effects on the subgingival microflora of acrylic strips containing $40 \%$ chlorhexidine, metron- idazole or tetracycline. $J$ Clin Periodontol 11: 379-386.

13. Goodson, J. M., Hogan, P. E. \& Dunham, S. L. 1985. Clinical responses following periodontal treatment by local drug delivery. J Periodontol 56 (Suppl. 11): 81-87.

Address:

W. J. Loesche

University of Michigan

School of Dentistry

Ann Arbor

Michigan 48109

U.S.A. 
This document is a scanned copy of a printed document. No warranty is given about the accuracy of the copy. Users should refer to the original published version of the material. 